\title{
羧甲基纤维素锂(CMC-Li)的合成流变和静电纺丝功能化材料研究
}

\author{
邱䂞 ${ }^{a}$ 邵自强*, ${ }^{a}$ 王建全 ${ }^{a}$ 张大伦 ${ }^{a}$ 曹军 ${ }^{b}$ \\ ( ${ }^{a}$ 北京市纤维素及其衍生物材料工程技术研究中心 北京理工大学材料学院 北京 100081) \\ ( ${ }^{b}$ 西安北方惠安化学工业有限公司 西安 710302)
}

\begin{abstract}
摘要 对离子型纤维素醚静电纺丝研究依旧是当前研究的难点. 研究表明, 成功合成新的离子型纤维素醚 CMC- $\mathrm{Li}$, 并 对比羧甲基纤维钠 $(\mathrm{CMC}-\mathrm{Na}$ )进行流变测试, 两者都表现出非牛顿流体的特性, 且取代度和黏度相近的 CMC-Li 比 $\mathrm{CMC}-\mathrm{Na}$ 具有更低的非牛顿指数, 对于同类物质低取代度、高浓度的溶液表现出更低的非牛顿指数. 并针对聚合物的结 构特点, 易与水溶性端羟基高分子量的聚环氧乙烷(PEO)配成静电纺丝优良共纺溶液, 当速率为 $4 \mathrm{~mL} / \mathrm{h}$, 电压为 $25 \mathrm{kV}$, 纺丝距离为 $12 \mathrm{~cm}$ 时能得到外观形貌较好的平均直径为 $70 \mathrm{~nm}$ 左右的纳米纤维. CMC-Li 具有更好的纺丝性能, 对 CMC-Li 包覆碳化功能化复合材料研究进行初步的探讨. 通过使用红外(IR)、流变仪、扫描电镜(SEM)分析取得较好的 效果.
\end{abstract}

关键词 静电纺丝; 纤维素及其衍生物; 流变性能; CMC-Li; 功能材料

\section{Study on Synthesis, Rheological and Electrospinning Functional Materials of Carboxymethyl Cellulose Lithium (CMC-Li)}

\author{
Qiu, Lei ${ }^{a} \quad$ Shao, Ziqiang*,a Wang, Jianquan ${ }^{a} \quad$ Zhang, Dalun $^{a} \quad$ Cao, Jun ${ }^{b}$ \\ $\left({ }^{a}\right.$ Beijing Engineering Research Center of Cellulose and Its Derivatives, School of Materials Science and Engineering, \\ Beijing Institute of Technology, Beijing 100081) \\ ( ${ }^{b} X i$ 'An North Hui An Chemical Industries CO., LTD, Xi'An 710302)
}

\begin{abstract}
The study on electrospinning of natural polysaccharide polymer to be nano-crystallized is still difficult in the current time. Carboxymethyl cellulose sodium (CMC-Na) was successfully synthesized and acidized to obtain carboxymethyl cellulose hydrogen (CMC-H), and new ionic cellulose ether CMC-Li was synthesized by alkalization, and then we analyzed the difference of structure between the three products. By comparing the rheological measurement and analysis of CMC-Na and CMC-Li, which have different mass fractions, it is found that they both show the characteristics of non-Newtonian fluid. The non-Newtonian index of CMC-Li is lower than that of CMC-Na when the degree of substitution (DS) and viscosity of both is similar. For the same material, the ionic cellulose ether solution of lower DS and higher concentration shows the lower non-Newtonian index. For the structure and property characteristic of polymers, it was easy to combine them with water-soluble hydroxyl terminated high molecular weight polyethylene oxide (PEO) to allocate the excellent electrospinning solution. Processing conditions were adjusted to a flow rate of $4 \mathrm{~mL} / \mathrm{h}$, an applied voltage of $25 \mathrm{kV}$, the spinning distance of $12 \mathrm{~cm}$, the better appearance of nanofibers whose average diameter is about $70 \mathrm{~nm}$ can be obtained. In the process of electrospinning, hydroxyl lithium ester enolates was obtained by reductive reaction of the ionized $\mathrm{Li}^{+}$with active hydroxyl, which caused the molecular structure to be destroyed. CMC-Li showed the exceptional Non-Newtonian fluid properties and had the better spinning performance than CMC-Na. When the surface tension of liquid was damaged by external electric force of spinning voltage to balance each other, the obtained morphology of the fiber was better. Nano-aluminum powder is coated with CMC-Li to form composite nanofibers, and carbonized at the high temperatures. $\mathrm{Al} / \mathrm{CNF} / \mathrm{Li}$ composite fiber was obtained in which nano particles were uniformly distributed. We preliminarily studied the obtained composite material that remained morphology and structure of fiber, which lays the foundation for the later research that the material is applied to soluble membrane, tissue engineering scaffolds, biological medicine, lithium battery and super capacitor, etc. The satisfactory effects have been achieved through analysis of IR, Rheology and SEM. The paper expands the type of polysaccharide polymer that can be electrospun and filled the void of related research, and it also lays the foundation for the study on nano functional materials of new ionic cellulose ether.
\end{abstract}

Keywords electrospinning; cellulose and its derivatives; rheological properties; CMC-Li; functional materials

\footnotetext{
*E-mail: shaoziqiang@263.net

Received June 28, 2013; published August 14, 2013.
}

Supporting information for this article is available free of charge via the Internet at http://sioc-journal.cn.

Project supported by the the National Science-technology support plan projects (No. 2011BAD23B04), special programs of graduate students' scientific and technological innovative activities of Beijing institute of technology (No. 3090012241302) and Youth Science and Technology Innovation Projects of North Chemical Industry CO., LTD (No. QKCZ-BIT-04).

受 “十二五” 国家科技支撑计划项目(No. 2011BAD23B04), 北京理工大学研究生科技创新基金(No. 3090012241302)和北方工业集团青年科技创新专 项基金(No. QKCZ-BIT-04)资助. 


\section{1 引言}

静电纺丝法 ${ }^{[1]}$ 是一种高效制备纳米纤维的技术, 形 成非牛顿流体的高聚物或熔融体在突破高压静电力的 作用下, 形成纳米纤维, 只有具有非稳定性的非牛顿流 体才能进行静电纺丝, 这是该方法的基本特征之一. 不 仅可将多种合成和天然聚合物电纺成单一纳米纤维 ${ }^{[2,3]}$, 还可以得到复合纳米纤维材料, 例如同轴纺 丝 $^{[4]}$ 、三轴 纺丝 ${ }^{[5,6]}$ 、多层交叉纺丝等 ${ }^{[7 \sim 9]}$ 技术的应用, 推动了该技 术的快速发展. 由于纳米纤维材料性能的优良特性, 在 膜过滤器 ${ }^{[10,11]}$ 、组织工程支架 ${ }^{[12,13]}$ 、生物工程 ${ }^{[14]}$ 、临床 医学 ${ }^{[15,16]}$ 、超敏传感器 ${ }^{[17,18]}$ 、锂电池材料 ${ }^{[19]}$ 和超级电容 器 ${ }^{[20]}$ 等方面具有巨大的研究价值. 然而, 目前能进行静 电纺丝的常用聚合物种类还相对较少, 主要集中在聚乙

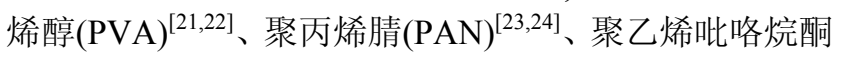

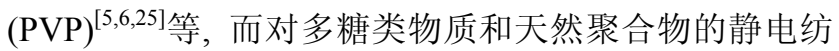

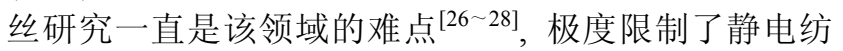
丝研究的进一步发展.

纤维素及衍生物 ${ }^{[2,30]}$ 是最有规模前景的资源之一, 具有其他材料无法比拟的研究价值. 该类材料已经在静 电纺丝研究方面受到学者的广泛关注，通过不同的溶剂 体系研究, 主要集中在醋酸纤维素 $(\mathrm{CA})^{[31]}$ 、羟丙基纤维

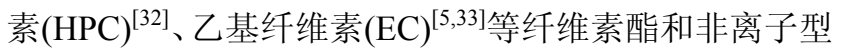
纤维素醚. 我们课题组一直致力于这方面的研究, 还在 纤维素、羟丙基甲基纤维素(HPMC) ${ }^{[34]}$ 、硝化纤维素(NC) 以及其衍生物 ${ }^{[35,36]}$ 、乙酸丁酸纤维素 $(\mathrm{CAB})$ 及其衍生 物 ${ }^{[37]}$ 等取得了较好的效果. 离子型纤维素醚由于它自 身结构和性能的原因，一直没有找到一种合适的电纺丝 途径而暂时没有被报道. 被称为 “工业味精” ${ }^{[38,39]}$ 的 CMC-Na 有着其他材料无法比拟的优点, 它成本低, 易 水溶、环境友好、工艺简单，广泛应用于石油、涂料、 食品、膜材料、陶瓷、医药、电池、航天航空材料等领 域. 关于相关的静电纺丝研究, 特别是 CMC-Li 的研究, 还暂未见有相关报道.

本文介绍合成新的离子型纤维素醚 CMC-Li 的方 法, 通过比较 CMC-Na 的结构分析与溶液流变性的差异 性研究, 进一步研究了各因素对其静电纺丝工艺的影 响. 还利用 CMC-Li 进行功能化包覆碳化形成复合材料 研究进行了探讨.

\section{2 结果与讨论}

\section{1 离子型纤维素醚的分子结构分析}

由图 1 中可知: 纤维素粉发生化学反应生成 CMC-Na, 但是 CMC-Li 的特征基团峰与 CMC-Na 的非 常接近, 只是峰强度随取代度的不同而有所差别, 而羧 甲基纤维素氢 (CMC-H) 的特征基团峰明显不同于 CMC-Na，说明酸化后的 CMC-Na 的分子结构发生了明 显变化, 而且 $\mathrm{CMC}-\mathrm{H}$ 不溶于水; 将 $\mathrm{CMC}-\mathrm{H}$ 置于坩埚中
于 $700{ }^{\circ} \mathrm{C}$ 下灼烧 $30 \mathrm{~min}$, 没有发现物质残留，说明酸化 后的 CMC-H 无钠残留. 除了羧甲基纤维素盐中共同结 构的特征峰外, 在 $723 \mathrm{~cm}^{-1}$ 处两种羧甲基纤维素盐之间 有较明显的区别, 也证明了 CMC-Li 的成功生成, CMC-H 和 CMC-Li 在 1757, 1602 和 $1424 \mathrm{~cm}^{-1}$ 处有很 大不同，这是因为羧酸基的酸性质子被 $\mathrm{Li}$ 离子取代后， 耦合在同一碳原子上的 $\mathrm{C}=\mathrm{O}$ 键和 $\mathrm{C}-\mathrm{O}$ 键被均化.

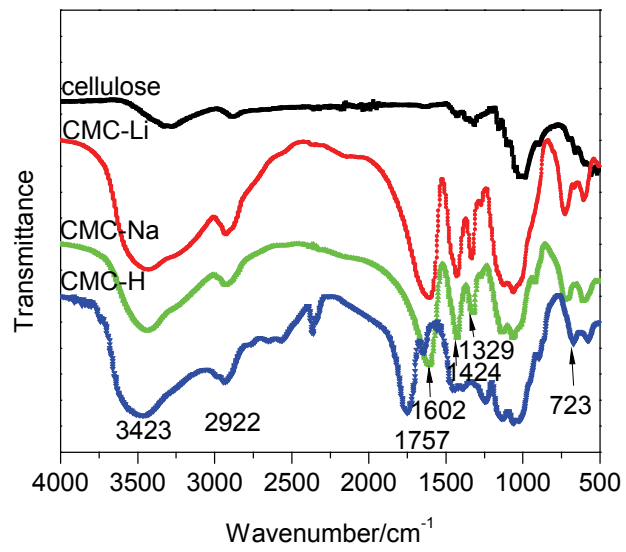

图 1 纤维素及其产品的红外结构分析

Figure 1 The infrared structure analysis of cellulose and its products

这两个被均化的碳氧键, 力常数的大小介于双键 $\mathrm{C}=\mathrm{O}$ 和单键 $\mathrm{C}-\mathrm{O}$ 之间，两个均等的碳一氧键的伸缩振 动发生强烈的耦合, 结果在 1602 和 $1424 \mathrm{~cm}^{-1}$ 出现两个 吸收带, 也证实 CMC-H 的生成. CMC-H 可作为重要的 中间产物来帮助判断纤维素粉, CMC-Na, CMC-H 和 CMC-Li 的生成结果.

CMC-Li 的 ${ }^{1} \mathrm{H}$ NMR 核磁谱图如图 2 所示. 右上角 插图是 CMC-Li 的结构示意图. 图 2 中化学位移 $\delta 0.7 \sim 1.6$ 之间的特征峰是由葡萄糖环上氢引起的, 理 论上积分总和对应葡萄糖环上的 6 个氢; 化学位移 $\delta$ $1.6 \sim 2.2$ 之间的峰对应的是 $\mathrm{CH}_{2} \mathrm{COO}^{-}$中 $\mathrm{H}$ 特征峰. $\mathrm{CH}_{2} \mathrm{COO}^{-}$在此区域产生了尖锐的强峰，从低场到高场 分别是 $\mathrm{C} 3, \mathrm{C} 2-\alpha, \mathrm{C} 2-\beta$ 和 C6 位上的质子峰; 化学位移 $\delta$ $2.2 \sim 3.1$ 之间的两组双重峰是由水解后葡萄糖单元 (AGU)上 $\mathrm{C} 1$ 位 $\mathrm{H}$ 引起的. 其中低场双重峰是 $\alpha$-异构体 上的 $\mathrm{H}$; 高场双重峰是 $\beta$-异构体上的 $\mathrm{H}$. 每组双重峰中

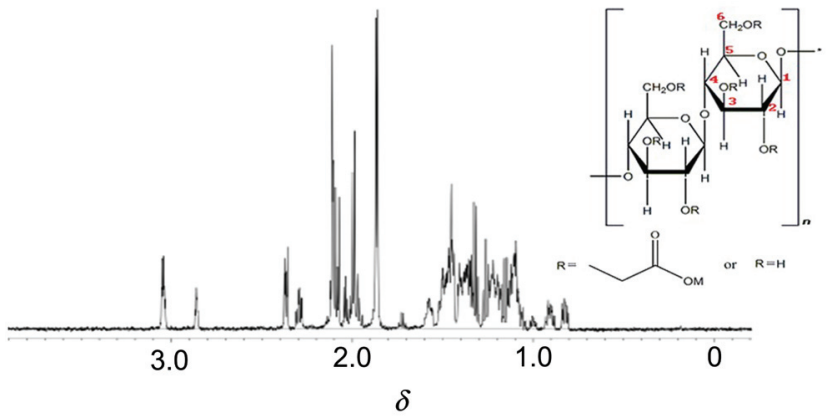

图 2 CMC-Li 的 ${ }^{1} \mathrm{H} \mathrm{NMR}$ 核磁谱图

Figure $2{ }^{1} \mathrm{H}$ NMR nuclear magnetic resonance spectrum of CMC-Li 
的两峰的区别在于低场峰是由于 $\mathrm{C} 2$ 位被羒甲基取代, 羧甲基上的 $\mathrm{H}$ 对 $\mathrm{C} 1$ 上 $\mathrm{H}$ 电子云产生吸引(向低场位移) 所引起的, 高场峰则说明了 $\mathrm{C} 2$ 的羟基未被取代.

综合上述分析, 说明 CMC-Na 经先酸化后中和的方 法可以得到水溶性的 CMC-Li. 并且从原料到产物, 取 代基团分布基本不变, 同时成功合成得到一种新的离子 型纤维素醚 CMC-Li.

\section{2 羧甲基纤维素盐的静电纺丝溶剂研究}

离子型纤维素醚的静电纺丝由于其特殊的分子结 构和性质，难以配制一种适合的静电纺丝液，一直是静 电纺丝领域的难点. 在大量实验的基础上, 利用水溶性 物质的特点, 添加共溶剂, 相互配制纺丝液, 得到纺丝 纤维后, 再除去共溶剂, 如表 1 和图 3 所示, 列举对比几 种常用的水溶性纺丝溶液, 将羧甲基纤维素盐(CMC-X, $X=\mathrm{Na}$ 或 $\mathrm{Li}$ ) 溶解其中, 发现只有当较高分子量的聚环 氧乙烷(PEO)为共溶剂时, 较容易得到可纺性较好的纺 丝溶液. 将一定质量分数的 CMC-X 溶解在相同质量分 数、不同分子量的 PEO 中, 只有 CMC-X 在分子量为 30 万(如图 A)和 40 万(如图 B)的两种 PEO 结构的溶剂中才 可以进行静电纺丝, 并且图 $\mathrm{B}$ 显示了能得到形貌更好的 $\mathrm{CMC}-\mathrm{X}$ 纳米纤维, 这可能是因为 $\mathrm{CMC}-\mathrm{X}$ 和 $\mathrm{PEO}$ 分子 端基位置都含有羟基, 与水结合以后, 由于不同位置的 羊基发生的位阻效应和亲核性的影响, 容易结合高分子 量的 PEO 形成良电纺共溶剂, 溶液非牛顿流体体系更 好, 改变了混合液的黏度和分子结构体系, 在静电力的 作用下, 更易突破静电力, 形成表面形貌更好的纳米纤 维.

表 1 几种常用的水溶性纺丝溶剂 ${ }^{a}$

Table 1 Several commonly used water-soluble spinning solvent

\begin{tabular}{cccc}
\hline 物质 & 共溶剂 & 常温可溶性 & 可纺性 \\
\hline CMC-X & PVP & 可溶 & 不可纺 \\
CMC-X & PEO (10 万) & 可溶 & 不可纺 \\
CMC-X & PEO (30 万) & 可溶 & 可纺 \\
CMC-X & PEO (40 万) & 可溶 & 可纺 \\
CMC-X & PVA & 可溶 & 不可纺 \\
CMC-X & THF & 不可溶 & 不可纺 \\
\hline${ }^{a}$ THF(聚四氢呋喃), CMC-X 为 CMC-Na 或 CMC-Li &
\end{tabular}

随后, 比较了不同比例的 CMC-X 和 PEO 溶于水中 直接进行静电纺丝, 分别选取了 $\mathrm{CMC}-\mathrm{X}: \mathrm{PEO}=0: 1$, $0.3: 1,0.5: 1,1: 1,2: 1,3: 1,1: 0$, 发现仅为纯的 CMC-X 或者 PEO 时, 都无法得到形貌较好的纺丝纤维, 其他比例的纺丝纤维经过萃取掉 PEO 以后, 烘干进行 SEM 表征, 发现形貌各异(如图 $\mathrm{C} \sim \mathrm{H}$ 所示), 通过比较 得到, 当 $C M C-X: P E O=1: 1 \sim 3: 1$ 时, 能帮助得到 形貌较好, 直径为 $70 \mathrm{~nm}$ 左右的纳米 CMC-X 纤维. 综合 考虑, 在后续实验中, 均选取分子量为 40 万的质量分数 为 $2 \%$ 的 PEO 作为共溶剂配制纺丝液.

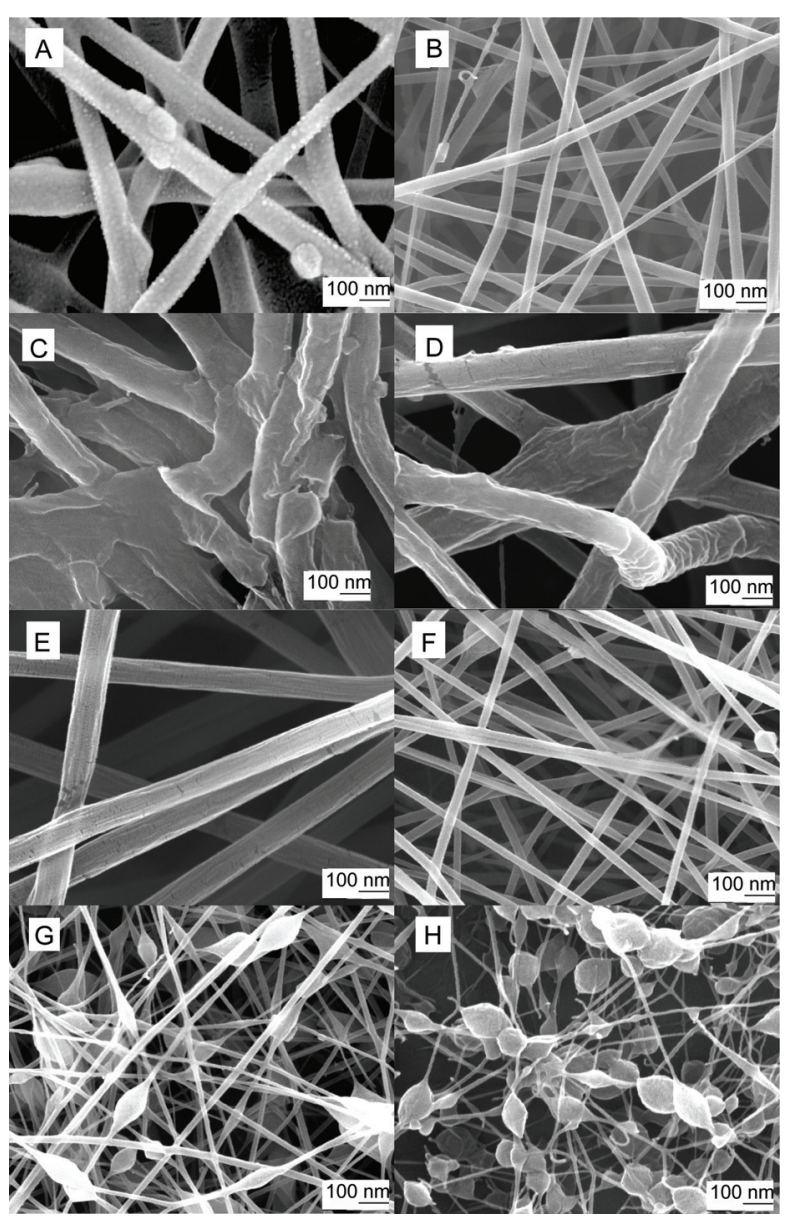

图 3 羧甲基纤维素盐的不同溶剂性能研究的 SEM 分析

Figure 3 The SEM analysis of different solvent properties of ionic cellulose ether

The PEO molecular weight of A is 300000 , the PEO molecular weight of $\mathrm{B}$ is 400000 , the mass ratio of $\mathrm{CMC}-\mathrm{X}$ and $\mathrm{PEO}$ of $\mathrm{C} \sim \mathrm{H}$ is $0.3: 1,0.5: 1,1: 1$, $2: 1,3: 1,4: 1$

\subsection{CMC-Na 和 CMC-Li 共纺液的流变性能分析}

图 4 为 $20{ }^{\circ} \mathrm{C}$ 下, 四种不同浓度, 相近取代度和黏 度的 CMC-Na 和 CMC-Li 分别与质量分数为 $2 \%$ 的 PEO 形成共纺液的流变曲线图, 其中 $\mathrm{CMC}-\mathrm{Na}$ 为合成 $\mathrm{CMC}-\mathrm{Li}$ 的原料. 从图中可以看出, CMC-Li 保持了与 $\mathrm{CMC}-\mathrm{Na}$ 水溶液一致的流变特性, 即其剪切黏度随剪切 速率的增大而降低, 整个流变曲线呈现典型假塑性流体 剪切稀释的非牛顿流体特征. 同时, 根据幂率公式 ${ }^{[40]}$ 可 以计算得到四种不同离子型纤维素醚不同浓度的非牛 顿流体指数 $n$ 值, 如表 2 所示, 发现相近取代度和黏度 的相同浓度的 CMC-Li 比 CMC-Na 具有更低的 $n$ 值, 可 能由于 CMC-Li 的分子链活动空间较小, 使松弛过程较 慢, 链段松弛时间增加, 传递能量减小, 溶液的假塑性 增加，在剪切应力的作用下分子链更容易定向、伸展和 解缠绕，从而表现出更多的非牛顿性. 在相同浓度下, 取代度低的高分子 $n$ 值较高取代度要小, 可能是因为低 取代度 CMC-Li 的刚性增强, 键结点和几何缠结点增加, 

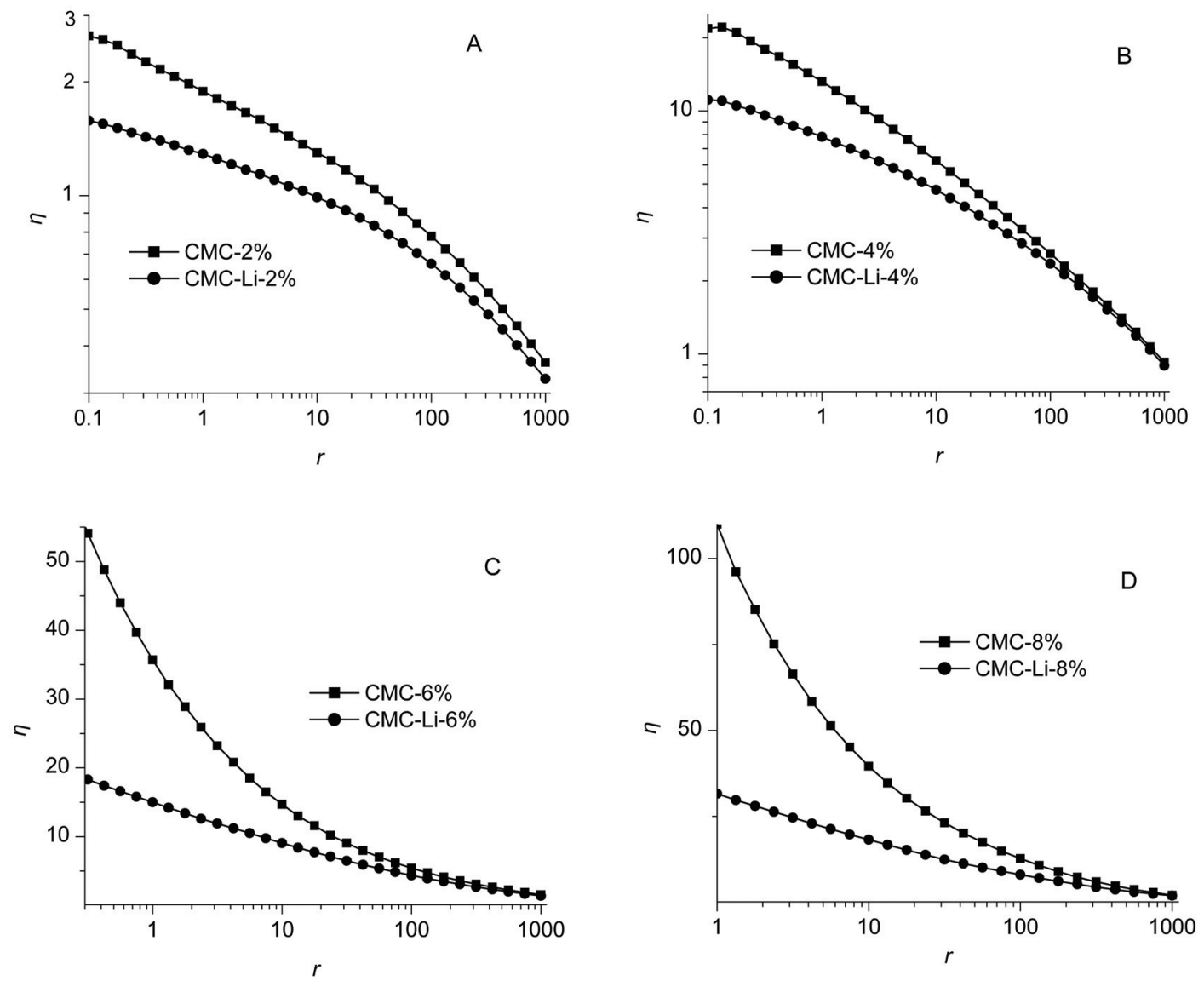

图 $4 \mathrm{CMC}-\mathrm{Na}$ 和 $\mathrm{CMC}-\mathrm{Li}$ 溶液的稳态流动曲线

Figure 4 Steady-state flow curve of CMC-Na and CMC-Li solution

表 2 不同物质不同浓度下的非牛顿指数

Table 2 Non Newton index under different concentrations of different substances

\begin{tabular}{cccccc}
\hline \multirow{2}{*}{ 物质种类 } & \multicolumn{5}{c}{$n$} \\
\cline { 2 - 6 } & $2 \%$ & $3 \%$ & $4 \%$ & $6 \%$ & $8 \%$ \\
\hline CMC-Na-1 & 0.89 & 0.88 & 0.81 & 0.81 & 0.79 \\
CMC-Na-2 & 0.97 & 0.96 & 0.93 & 0.87 & 0.83 \\
CMC-Li-1 & 0.84 & 0.78 & 0.72 & 0.64 & 0.59 \\
CMC-Li-2 & 0.98 & 0.93 & 0.91 & 0.87 & 0.78 \\
\hline
\end{tabular}

造成非牛顿指数减小. 同时, 随着纤维素醚含量的增加, 纺丝液中大分子间的缠结点浓度升高, 使黏度增大, 流 动性变差, 当剪切速率较大时, 大部分缠结点被破坏掉, 造成黏度快速下降, $n$ 值减小. 结合电纺的情况, 纤维素 醚的浓度控制在 3\% 6\%之间较合适.

\subsection{CMC-Na 和 CMC-Li 的静电纺丝研究}

在其他纺丝条件不变的情况下, 将相同质量、相同 取代度和黏度的 CMC-Na 和 CMC-Li 分别溶解在相同的 纺丝共溶剂中，以相同的纺丝条件得到不同形貌的纺丝 纤维, 如图 5 所示, $\mathrm{A} \sim \mathrm{C}$ 为 $\mathrm{CMC}-\mathrm{Na}$ 的纺丝纤维, $\mathrm{D} \sim \mathrm{F}$ 为 CMC-Li 的纺丝纤维, 从图中可以看出, 两者都得到

了较好形貌的离子型羧甲基纤维素醚纤维, 然而, CMC-Li 的表面更加光滑, 纤维直径分布更加均匀, 较 少出现珠节和断点的地方, 这可能是由于 CMC-Li 体现 出具有更好的非牛顿性能, 当与 PEO 形成共溶剂时, $\mathrm{CMC}-\mathrm{Li}$ 容易电离出 $\mathrm{Li}^{+}$, 易与活泼的羟基发生反应, 在 外电场静电纺丝过程中还原成烯醇锂 ${ }^{[4]}$ 结构 $\left(=\mathrm{C}-\mathrm{O}^{-}\right.$ $\mathrm{Li}^{+}$), 更有利于形成纺丝射流，分子链结构特征更易被 破坏, 发生构象变化, 开始解缠绕并沿着流动方向取向, 所以, CMC-Li 能得到更好形貌的纳米纤维.

\section{5 不同的电纺速率对电纺纤维形貌的影响}

在其他实验条件一定的情况下，我们研究了不同纺 丝速率对纤维形貌和直径的影响如图 6 所示. 随着速率 的增大, 得到的纤维的直径变化较小, 当纺丝速率过大 或者过小时, 纤维中的珠节、断丝现象和粘接现象会有 所增加, 这可能是由于改变挤出速率, 易在针口形成不 稳定的流量, 造成分子解缠绕后的流动速度和外电场力 破坏分子结构的速度的不均衡，同时也造成形成的泰勒 锥处的液体的表面张力和外电场力的不均衡, 影响纤维 的外观形貌和溶剂的挥发速度. 综合考虑, 采用纺丝速 率为 $4 \sim 6 \mathrm{~mL} / \mathrm{h}$ 时, 能得到外观形貌较好的纳米 $\mathrm{CMC}-\mathrm{Li}$ 纤维. 


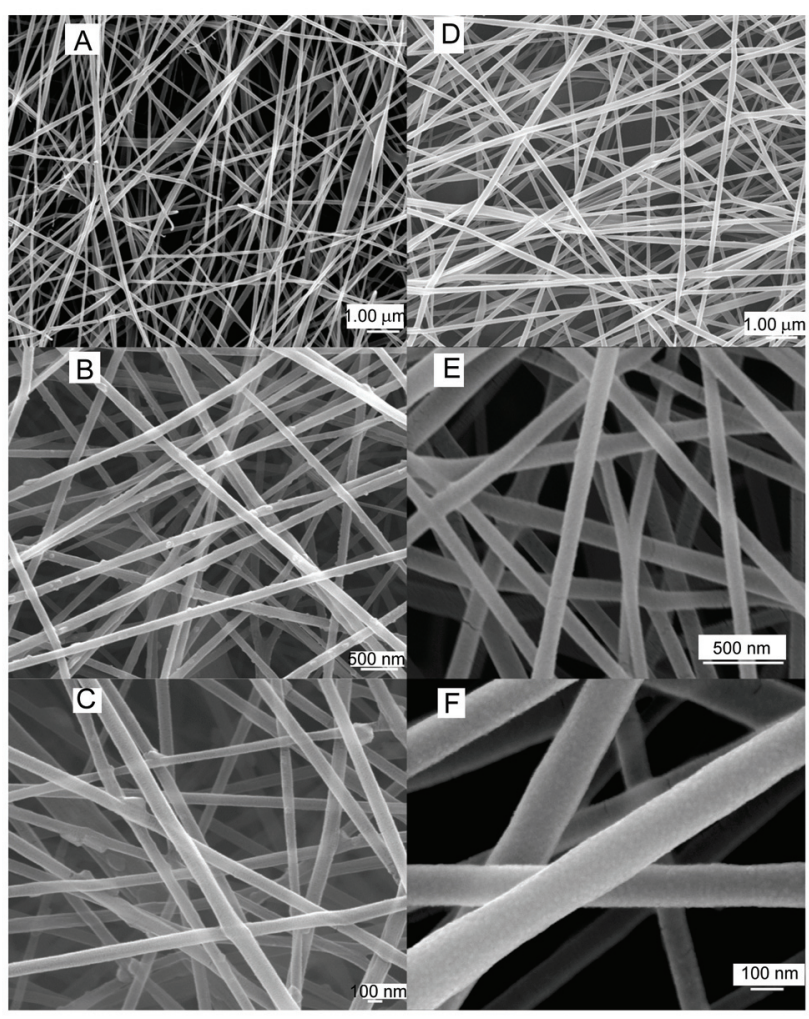

图 5 CMC-Na 和 CMC-Li 的静电纺丝研究的 SEM 分析

Figure 5 The SEM analysis of CMC-Na and CMC-Li by electrospinning

Spinning distance is $12 \mathrm{~cm}$, and flow rate is $4 \mathrm{~mL} / \mathrm{h}$ and voltage is $25 \mathrm{kV}$.

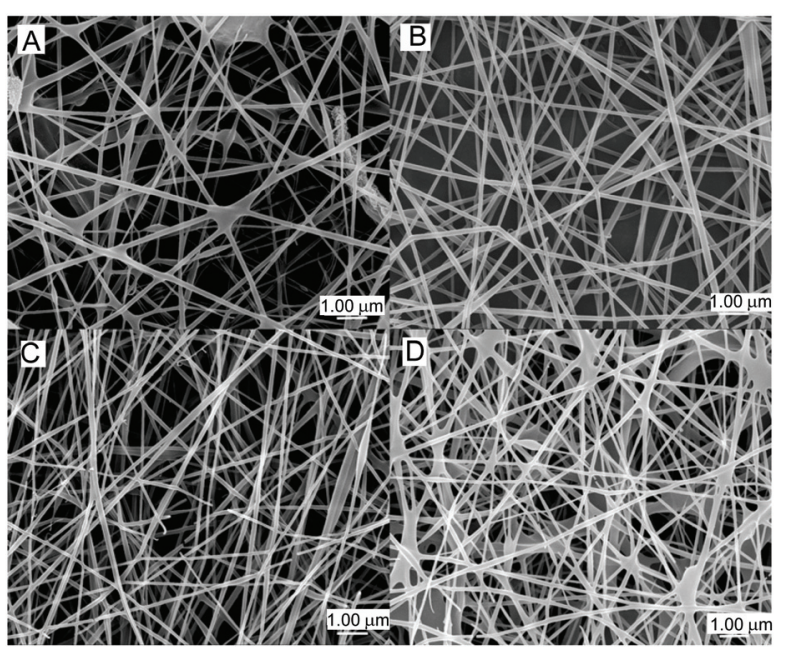

图 6 不同挤出速率下 CMC-Li 的电纺纤维 SEM 形貌

Figure 6 The SEM morphology of electrospun CMC-Li fiber at different primary rate

The primary rate of $\mathrm{A} \sim \mathrm{D}$ is: $2,4,6,8 \mathrm{~mL} / \mathrm{h}$

\subsection{CMC-Li 包覆复合材料的功能化研究}

在 CMC-Li 的电纺溶液中加入一定量的纳米金属铝 粉, 超声分散完全后, 悬浮电纺液中, 再按照静电纺丝
法得到萃取后的干燥纳米纤维. 从图 7 中 A、B 可以看 出纤维直径较小 (约 $70 \mathrm{~nm}$ ) 且均一, 纳米铝粉在 $\mathrm{CMC}-\mathrm{Li}$ 纤维基体中分散比较均匀. 这可能是由于在静电纺丝过 程中纳米铝粉带有同种电荷, 相互排斥作用使其在电纺 的过程中沿纤维的轴向和径向两个方向进行分散，由于 纤维的直径较小, 径向空间较小, 沿纤维径向的分散受 到空间位阻的影响，而纤维长度较大，轴向空间较大， 沿轴向分散容易且空间位阻小，结果是纳米粒子沿轴向 均匀分散. 将得到的纤维在一定温度下进行碳化处理, 如图 7 中 $\mathrm{C}, \mathrm{D}$ 所示, 得到保持较好纤维结构的 $\mathrm{Al} / \mathrm{C} / \mathrm{Li}$ 复合材料. 可见静电纺丝技术能较好地解决纳米粒子之 间的团聚问题，也为以离子型纤维素醚作为静电纺丝载 体进行包覆或者进而其他功能化的研究奠定基础, 扩大 了静电纺丝聚合物的范围, 也为离子型纤维素醚的纳米 功能化研究奠定了新的基础.

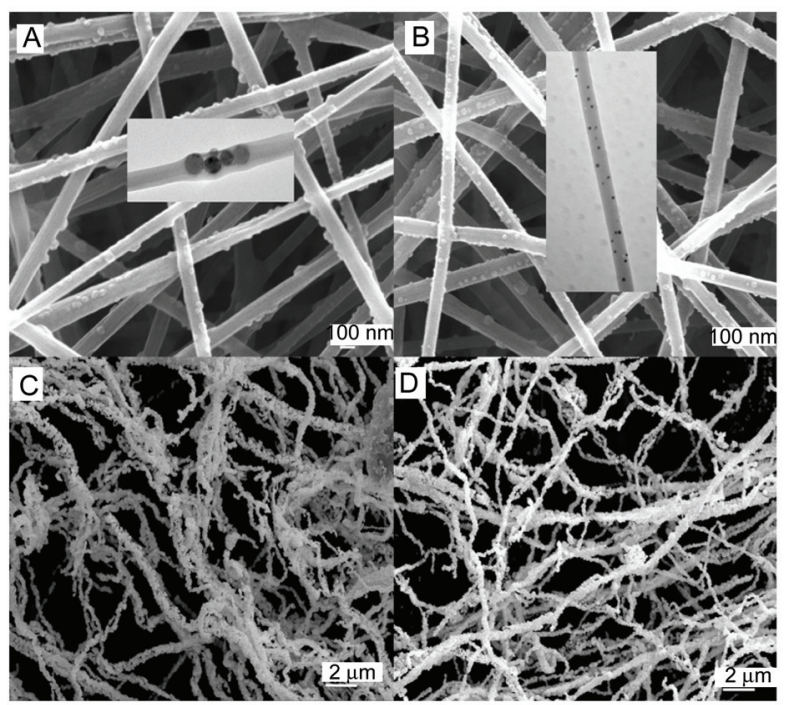

图 7 以 CMC-Li 作为载体包覆纳米金属粒子研究的 SEM 照片 Figure 7 The SEM analysis of regarding CMC-Li as a carrier to coat metal particles (A, B before carbonization, C, D after carbonization)

\section{3 结论}

成功合成一种新的离子型纤维素醚 CMC-Li, 并研 究了不同常用溶剂溶解 CMC-X 与静电纺丝之间的机理, 并从分子基团结构层面解释了原因. 进行流变性能分 析，发现 CMC-Li 比 CMC-Na 具有更好的非牛顿流体特 性, 并首次通过静电纺丝法得到纳米的 $\mathrm{CMC}-\mathrm{Li}$ 纤维, 且具有更好的电纺性. 对 CMC-Li 作为载体, 碳化, 得 到形貌较好的复合纳米功能化纤维材料进行探索, 为纳 米纤维的功能化研究奠定基础.

\section{4 实验部分}

\section{1 合成 CMC-Li}

CMC-Li 的合成分为两步: 


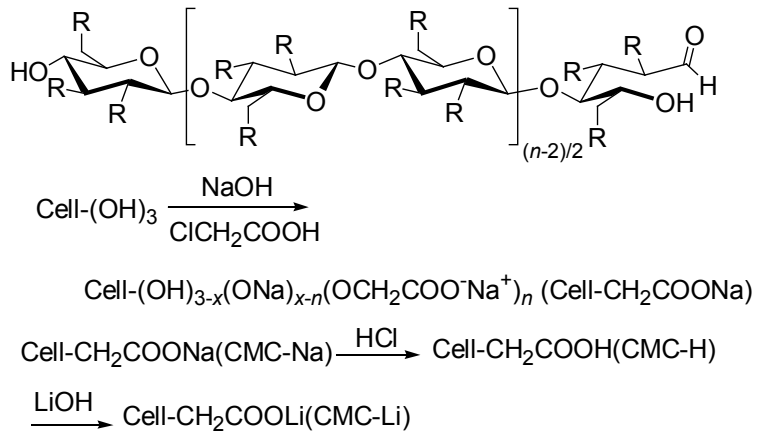

图 8 目标化合物的合成路线 $\left[\mathrm{R}=\mathrm{OH}, \mathrm{Cell}-(\mathrm{OH})_{3}\right]$

Figure 8 The synthetic route of the target compounds $[\mathrm{R}=\mathrm{OH}$, Cell- $\left.(\mathrm{OH})_{3}\right]$

\subsection{1 原料精制棉的千燥处理及 CMC- $\mathrm{Na}$ 制备 ${ }^{[29]}$ \\ 4.1.2中间产物 CMC-H 的合成}

以 $95 \%$ 的酒精 (体积分数) 作为分散介质, 将 CMC-Na 分散其中, 摚拌下加入一定量的盐酸(用量依 据原料 $\mathrm{CMC}-\mathrm{Na}$ 的取代度而定), 维持温度在 $35{ }^{\circ} \mathrm{C}$, 反 应 $2 \mathrm{~h}$. 反应结束后过滤, 用 $85 \%$ 乙醇溶液洗涤两遍, 最 后在 $105{ }^{\circ} \mathrm{C}$ 下干燥, 粉碎得 CMC-H.

\subsection{3 羒甲基纤维素锂盐的制备}

对于 CMC-Li 制备, 分为两步法: 即 CMC-Na 先酸 化再碱化. (1) CMC-H 的制备. (2)将纯净的 CMC-H 分散 在 $90 \%$ 的酒精、水溶液中, 加入一定量的氢氧化锂水溶 液(浓度根据取代度不同而定), 维持温度在 $50{ }^{\circ} \mathrm{C}$, 反应 $2 \mathrm{~h}$, 反应后冰醋酸中和至中性，过滤，用 $85 \%$ 乙醇溶液 洗涤数遍, 最后在 $105{ }^{\circ} \mathrm{C}$ 下干燥 $2 \mathrm{~h}$, 粉碎得羧甲基纤 维素锂. 所得产物分别为: CMC-Na-1, 取代度 $\mathrm{DS}=$ 0.83 , 黏度 $\eta=143 \mathrm{mPa} \cdot \mathrm{s} ; \mathrm{CMC}-\mathrm{Na}-2, \mathrm{DS}=1.22, \eta=75.2$ $\mathrm{mPa} \bullet \mathrm{s} ; \mathrm{CMC}-\mathrm{Li}-1, \mathrm{DS}=0.86, \eta=141 \mathrm{mPa} \cdot \mathrm{s} ; \mathrm{CMC}-\mathrm{Li}-2$, $\mathrm{DS}=1.21, \eta=72.6 \mathrm{mPa} \cdot \mathrm{s}$.

\section{2 静电纺丝过程}

将 CMC-Na 和 CMC-Li 分别溶解在一定质量的水 中, 加入少量的 PEO $(c=2 \mathrm{wt} \%)$, 配制成一定浓度的纺 丝溶液, 装入挤出管中, 在挤出速率为 $4 \mathrm{~mL} / \mathrm{h}$, 挤出电 压为 $25 \mathrm{kV}$, 纺丝距离为 $12 \mathrm{~cm}$, 温度控制在 $25^{\circ} \mathrm{C}$ 左右 时, 进行静电纺丝, 得到纳米的离子型纺丝纤维, 将得 到的纳米纤维用加速溶剂萃取器将 PEO 用乙醇萃取除 去, 烘干纤维, 装袋备用.

\section{References}

[1] Liu, H.; Hsieh, Y. L. J. Polym. Sci., Part B: Polym. Phys. 2002, 40, 2119.

[2] Frey, M.; Joo, Y.; Kim, C. W. Polym. Prepr. (Am. Chem. Soc., Div. Polym. Chem.) 2003, 44, 168.

[3] Zhao, S.; Wu, X.; Wang, L.; Huang, Y. Cellulose 2003, 10, 405.

[4] Yu, D. G.; Wang, X.; Li, X. Y.; Chian, W.; Li, Y.; Liao, Y. Z. Acta Biomater. 2013, $9,5665$.

[5] Shi, Y.; Zhang, J.; Xu, S.; Dong, A. J. J. Biomater. Sci., Polym. Ed. 2013, 24,551

[6] Xu, Y.-Z.; Dong, X.-T.; Gai, G.-Q.; Wang, J.-X.; Liu, G.-X.; Lu,
T.-X. Acta Chim. Sinica 2012, 70, 1660. (徐淑芝, 董相廷, 盖广清, 王进贤, 刘桂霞, 鲁统晓, 化学学报, 2012, 70, 1660.)

[7] Li, H.; Shen, L.; Zhang, X.; Nie, P.; Chen, L.; Xu, K. J. Electrochem. Soc. 2012, 159, A426.

[8] Kong, J.; Liu, Z.; Yang, Z.; Tan, H. R.; Xiong, S.; Wong, S. Y.; Li, $\mathrm{X}$.; Lu, X. Nanoscale 2012, 4, 525.

[9] Hwang, T. H.; Lee, Y. M.; Kong, B.-S.; Seo, J.-S.; Choi, J. W. Nano Lett. 2012, 12, 802 .

[10] Zhang, J.; Liu, Z.; Kong, Q.; Zhang, C.; Pang, S.; Yue, L.; Wang, X.; Yao, J.; Cui, G. ACS Appl. Mater. Interfaces 2013, 5, 128.

[11] Li, Y.; Jiang, Y.; Liu, F.; Ren, F.; Zhao, G.; Leng, X. Food Hydrocolloid 2011, 25, 1098.

[12] Luo, Y.; Wang, S.; Shen, M.; Qi, R.; Fang, Y.; Guo, R.; Cai, H.; Cao, X.; Tomas, H.; Zhu, M.; Shi, X. Carbohydr. Polym. 2013, 91, 419.

[13] Nhu-Ngoc, B.; McCutcheon, J. R. Environ. Sci. Technol. 2013, 47, 1761.

[14] Manzine Costa, L. M.; de Olyveira, G. M.; Cherian, B. M.; Leao, A. L.; de Souza, S. F.; Ferreira, M. Ind. Crop. Prod. 2013, 41, 198.

[15] Nagy, Z. K.; Balogh, A.; Dravavoelgyi, G.; Ferguson, J.; Pataki, H.; Vajna, B.; Marosi, G. J. Pharm. Sci. 2013, 102, 508.

[16] Olaru, N.; Olaru, L.; Tudorachi, N.; Dunca, S.; Pintilie, M. Ind. Eng. Chem. Res. 2013, 52, 696.

[17] Ongun, M. Z.; Ertekin, K.; Hizliates, C. G.; Oter, O.; Ergun, Y.; Celik, E. Sens. Actuators B-Chem. 2013, 181, 244.

[18] Webster, M.; Miao, J.; Lynch, B.; Green, D. S.; Jones-Sawyer, R.; Linhardt, R. J.; Mendenhall, J. Macromol. Mater. Eng. 2013, 298, 447.

[19] Qiu, L.; Shao, Z.; Yang, M.; Wang, W.; Wang, F.; Xie, L.; Lv, S.; Zhang, Y. Carbohydr. Polym. 2013, 96, 240.

[20] Kang, Y. J.; Chun, S.-J.; Lee, S.-S.; Kim, B.-Y.; Kim, J. H.; Chung, H.; Lee, S.-Y.; Kim, W. ACS Nano 2012, 6, 6400.

[21] Lalia, B. S.; Samad, Y. A.; Hashaikeh, R. J. Solid State Electrochem. 2013, 17, 575.

[22] YerriSwamy, B.; Prasad, C. V.; Reedy, C. L. N.; Mallikarjuna, B.; Rao, K. C.; Subha, M. C. S. Cellulose 2011, 18, 349.

[23] Huang, X.-W.; Deng, J.-Y.; Xu, L.; Shen, P.; Zhao, B.; Tan, S.-T. Acta Chim. Sinica 2012, 70, 1604. (黄先威, 邓继勇, 许律, 沈平, 赵斌, 谭松庭, 化学学报, 2012, 70, 1604.)

[24] Prasanth, R.; Aravindan, V.; Srinivasan, M. J. Power Sources 2012 $202,299$.

[25] Wang, Y.-H.; Wang, J.-X.; Dong, X.-T.; Yu, W.-S.; Liu, G.-X. Acta Chim. Sinica 2012, 70, 1576. (王荣熇, 王进贤, 董相廷, 于文生, 刘桂霞, 化学学报, 2012, 70, 1576.)

[26] Awal, A.; Sain, M. J. Polym. Environ. 2012, 20, 690

[27] Gao, Y. F.; Yuan, J. Y.; Sui, X. F.; Zhou, M.; Cai, Z. N. Prog. Chem. 2009, 21, 1553.

[28] Frey, M. W. Polym. Rev. 2008, 48, 378.

[29] Cui, M.; Wang, F.-J.; Shao, Z.-Q.; Lu, F.-S.; Wang, W.-J. Cellulose 2011, 18, 1265

[30] Yang, Z. Y.; Wang, W. J.; Shao, Z. Q.; Zhu, H. D.; Li, Y. H.; Wang, F. J. Cellulose 2013, 20, 159.

[31] Konwarh, R.; Karak, N.; Misra, M. Biotechnol. Adv. 2013, 31, 421.

[32] Chang, C.; Zhang, L. Carbohydr. Polym. 2011, 84, 40.

[33] Kacmaz, S.; Ertekin, K.; Gocmenturk, M.; Suslu, A.; Ergun, Y.; Celik, E. React. Funct. Polym. 2013, 73, 674

[34] Ma, G.; Shao, Z. Q.; Wang, W. J.; Wang, F. J.; Tan, L. L.; Liao, B. Sci. China. Chem. 2010, 53, 190.

[35] Xie, L.; Shao, Z.; Wang, W.; Wang, F. Integr. Ferroelectr. 2011, $127,184$.

[36] Lu, S.-Y.; Shao, Z.-Q.; Zhang, Z.-L.; Wang, H.-Q. Acta Chim. Sinica 2012, 70, 200. (吕少一，邵自强，张振玲，王慧庆，王文俊， 化学学报, 2012, 70, 200.)

[37] Qiu, L.; Shao, Z.; Yang, M.; Wang, W.; Wang, F.; Li, Y.; Chen, J. Adv. Mater. Res. 2013, 634-638, 2613.

[38] Zhao, T.; Liu, L.; Li, G.; Du, L.; Zhao, X.; Yan, J.; Cheng, Y.; Dang, A.; Li, T. Chin. Sci. Bull. 2012, 57, 1620.

[39] Wang, Z.; Dupre, N.; Gaillot, A.-C.; Lestriez, B.; Martin, J.-F.; Daniel, L.; Patoux, S.; Guyomard, D. Electrochim. Acta 2012, 62, 77.

[40] Reneker, D. H.; Yarin, A. L. Polymer 2008, 49, 2387.

[41] Wang, W.-K.; Zhang, Y.; Wang, A.-B.; Yu, Z.-B.; Han, M.-F.; Yang, Y.-S. Acta Phys.-Chim. Sin. 2010, 26, 47. (王维坤, 张勇, 王 安邦, 余仲宝, 韩敏芳, 杨裕生, 物理化学学报, 2010, 26, 47.)

(Zhao, X.) 